\title{
Age-related alterations in inhibitory control investigated using the minimally delayed oculomotor response task
}

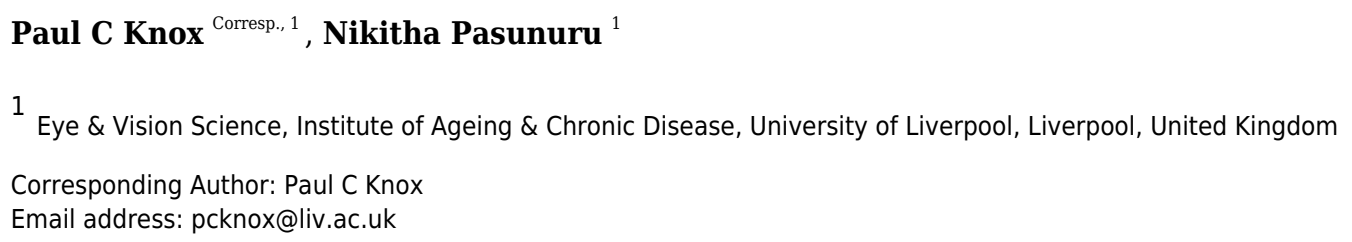

Healthy, older adults are widely reported to experience cognitive decline, including impairments in inhibitory control. However, this general proposition has recently come under scrutiny because ageing effects are highly variable between individuals, are task dependent, and are sometimes not distinguished from general age-related slowing. We recently developed the minimally delayed oculomotor response (MDOR) task in which participants are presented with a simple visual target step, and instructed to saccade not to the target when it appears (a prosaccade response), but when it disappears (i.e. on target offset). Varying the target display duration prevents offset timing being predictable from the time of target onset, and saccades prior to the offset are counted as errors. A comparison of MDOR task performance in a group of 22 older adults (mean age 62y, range $50 y$ to $72 y$ ) with that in a group of 39 younger adults (22y, range 19y-27y) demonstrated that MDOR latency was significantly increased in the older group by $34-68 \mathrm{~ms}$ depending on target display duration. However, when MDOR latencies were corrected by subtracting the latency observed in a standard prosaccade task, the latency difference between groups was abolished. There was a larger latency modulation with target display duration in the older group which was observed even when their generally longer latencies were taken into account. Error rates were significantly increased in the older group. An analysis of the timing distribution of errors demonstrated that most errors were failures to inhibit responses to target onsets. When error distributions were used to isolate clear inhibition failures from other types of error, the older group still exhibited significantly higher error rates as well as a higher residual error rate. Although MDOR latency in older participants may largely reflect a general slowing in the oculomotor system with age, both the latency modulation and error rate results are consistent with an age-related inhibitory control deficit. How this relates to performance on other inhibitory control tasks remains to be investigated. 
1

2

3

4

5

6

7

8 9

10

11

12

13

14

15

16

17

18

19

20

21

22

23

24

25

26

27

28

29

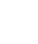

\author{
Paul C. Knox, Nikitha Pasunuru
}

\section{Age-related alterations in inhibitory}

control investigated using the minimally delayed oculomotor response task

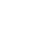

Eye and Vision Science, Institute of Ageing and Chronic Disease, University of Liverpool, Liverpool, UK.

\section{ORCID ID:}

Knox: 0000-0002-2578-7335

Corresponding Author:

Dr Paul C. Knox

Eye \& Vision Science

Institute of Ageing \& Chronic Disease

University of Liverpool

Liverpool

United Kingdom

Tel: 01517949042

pcknox@liv.ac.uk 
30

31

32

33

34

\section{Abstract}

Healthy, older adults are widely reported to experience cognitive decline, including impairments in inhibitory control. However, this general proposition has recently come under scrutiny because ageing effects are highly variable between individuals, are task dependent, and are sometimes not distinguished from general age-related slowing. We recently developed the minimally delayed oculomotor response (MDOR) task in which participants are presented with a simple visual target step, and instructed to saccade not to the target when it appears (a prosaccade response), but when it disappears (i.e. on target offset). Varying the target display duration prevents offset timing being predictable from the time of target onset, and saccades prior to the offset are counted as errors. A comparison of MDOR task performance in a group of 22 older adults (mean age 62y, range 50y to $72 y$ ) with that in a group of 39 younger adults (22y, range $19 y-27 y$ ) demonstrated that MDOR latency was significantly increased in the older group by $34-68 m s$ depending on target display duration. However, when MDOR latencies were corrected by subtracting the latency observed in a standard prosaccade task, the latency difference between groups was abolished. There was a larger latency modulation with target display duration in the older group which was observed even when their generally longer latencies were taken into account. Error rates were significantly increased in the older group. An analysis of the timing distribution of errors demonstrated that most errors were failures to inhibit responses to target onsets. When error distributions were used to isolate clear inhibition failures from other types of error, the older group still exhibited significantly higher error rates as well as a higher residual error rate. Although MDOR latency in older participants may largely reflect a general slowing in the oculomotor system with age, both the latency modulation and error rate results are consistent with an age-related inhibitory control deficit. How this relates to performance on other inhibitory control tasks remains to be investigated. 
56

57

58

59

60

61

62

63

64

65

66

67

68

69

70

71

72

73

74

75

76

77

\section{Introduction}

The observation that as we age the performance of many tasks becomes more difficult, leads easily to the narrative of inevitable age-related decline. It is unsurprising that this includes the cognitive domain (see Salthouse 2012 for a general review). Executive functions are a set of related abilities by means of which thought and action are controlled. While there is debate about how they are best classified, one approach views them as being separable into three broad domains of task or set shifting, working memory functions and inhibition, based on performance across a range of tasks (Miyake \& Friedman 2012; Miyake et al. 2000). In this and other accounts, inhibition or inhibitory control is a key domain (Verbruggen et al. 2014). The cognitive decline associated with ageing, has been specifically linked to an age-related deficit in inhibition (Hasher \& Zacks 1988). However, the hypothesis of a general inhibitory control deficit in older age, particularly once age-related changes in processing speed have been accounted for, is also matter of ongoing debate (Rey-Mermet \& Gade 2017; Rey-Mermet et al. 2018; Verhaeghen 2011; Verhaeghen 2014).

Inhibition itself is not a unitary concept and, as with executive function more generally, a number of taxonomies have been proposed to describe it (Rey-Mermet et al. 2018). Behavioural inhibition, often investigated with tasks where a motor response has to be prevented or stopped, is a prominent feature of most of these taxonomies. Some tasks used to investigate behavioural inhibition rely on manual responses (eg manual stop signal reaction time and go/no go tasks; Donders 1969; Logan \& Cowan 1984; Verbruggen \& Logan 2008), while others involve eye movements (eg saccade countermanding and no go tasks; Crawford et al. 2005; Hanes \& Carpenter 1999). Note that while these all involve aspects of motor behaviour, a central claim is that this general approach provides a means of exploring cognitive processing related to the control of thought as well as action (Castiglione et al. 2019; Logan \& Cowan 1984). 
84 A particular eye movement task, the antisaccade (AS) task (Hallett 1978; Hutton \& Ettinger 2006; Munoz \& Everling 2004), has been widely used in studies of executive function (Mirsky et al. 2011; Miyake \& Friedman 2012; Miyake et al. 2000), healthy ageing (Fernandez-Ruiz et al. 2018; Munoz et al. 1998; Olincy et al. 1997; Pa et al. 2014) and to investigate the effect of degenerative neurological conditions associated with ageing (Crawford et al. 2005; Kaufman et al. 2012). In many studies, the explicit motivation for using the AS task is to investigate inhibitory control (Alichniewicz et al. 2013; Baird-Gunning \& Lueck 2018; Crawford et al. 2002; Crawford et al. 2005; Diarra et al. 2019; Lennertz et al. 2012). In the AS task, a target appears to the left or right of fixation, but participants are instructed to look to the mirror image position of that target position (ie to direct their saccade in the opposite direction but the same distance from fixation). The directional error rate (the proportion of trials in which participants look at the target rather than to the instructed position) is taken to provide a measure of inhibition, with high error rates implying poor inhibitory control.

However, the view that increased AS directional error rate necessarily indicates an inhibitory control deficit is problematic. While successful AS performance requires the inhibition of the normal, reflexive, saccade response to a suddenly appearing target, it also requires the computation and execution of a voluntary saccade to an instructed position. This entails a competition for behavioural expression between two distinct behaviours (the reflexive prosaccade and the voluntary antisaccade), often modelled as a race between two signals rising to a triggering threshold (Munoz \& Everling 2004; Noorani \& Carpenter 2013). As whichever process reaches the trigger threshold first "wins" the race, any difficulty generating the voluntary saccade response will bias the competition in favour of the reflexive prosaccade (error) response, leading to a higher error rate. However, in this case, the increased error rate does not indicate a problem with inhibitory processing (Reuter et al. 2005). Allied to this, there is the well-recognised issue of task contamination. All tasks engage multiple processes, and this is true of the antisaccade task which requires both attentional (Gaspelin \& Luck 2018) and working memory resources (Crawford et al. 2011; Eenshuistra et al. 2004), two other key components of executive function (see also Magnusdottir et al. 2019). 
114 In order to address some of these issues we developed the minimally delayed oculomotor 115 response (MDOR) task (Knox et al. 2018; Wolohan \& Knox 2014). The stimulus used is a target 116 step with randomised direction and timing; target display duration (TDD) is also varied.

117 Participants are instructed to execute a saccade to a target appearing on either left or right of 118 fixation, not when it appears (a reflexive prosaccade response), but when the target is 119 extinguished. Performance is measured using the error rate (the proportion of trials in which participants execute a target directed saccade prior to target offset) and the latency of correct responses. We demonstrated that saccade latency in the MDOR task was much longer than is consistent with simple prosaccade responses to target onsets, and that it is modulated by TDD. Latency with a short TDD of $200 \mathrm{~ms}$ was of the order of $400 \mathrm{~ms}$. We interpreted this as indicating that saccades in this condition were executed against a high level of inhibition, leading to the considerable increase in latency compared to a standard prosaccade response in which latency would be expected to be approximately $200 \mathrm{~ms}$. With a longer TDD of $1000 \mathrm{~ms}$, latency was approximately $300 \mathrm{~ms}$, still considerably longer than might be expected. We hypothesised that is was lower because when the offset does not occur quickly, the level of inhibition present when the offset does occur has reduced. These latency effects were of a magnitude that were inconsistent with other known effects such as preview effects, and the lower salience of target offsets compared to onsets.

MDOR error rates are similar to error rates in the AS task, and also modulated by TDD. Analysis of the timing of error responses demonstrated that most errors were consistent with unsuccessfully inhibited responses to target onsets. We have shown previously that AS and MDOR error rates do not correlate in healthy participants (Wolohan \& Knox 2014). Manipulating fixation conditions (using gap and overlap task versions) did not significantly alter error rates and latencies in the MDOR task (Knox et al. 2018), although it does in the AS task. The alterations observed in the AS error rate are due to shifting the relative performance of the two competing tasks in the AS context as fixation conditions are changed (Munoz \& Everling 2004). In the MDOR task there are no competing processes of this type, and we argued that this 
142 explained the lack of gap and overlap effects. While the MDOR task, like every task, is not free

143 of task contamination, the working memory component is greatly reduced compared to the AS

144 task. Although there is an instruction to be remembered (ie saccade to the offset not the onset

145 of the target), there is no need to remember a target position (the target is displayed until

146 shortly before the saccade is initiated). And there is no need to disengage attention from the

147 target position, perform a vector inversion, and shift it to a new location, as would be required

148 for a correct antisaccade.

149

150 All of the experiments we have conducted with the MDOR task so far have been on younger

151 participants. Across multiple groups of younger participants, we have observed performance to

152 be similar and consistent, noticeably so for error rate (Knox et al. 2018). To explore the MDOR

153 task further, and given the prominence of saccade tasks in both the ageing and inhibitory

154 control literatures, our aim in the current study was to investigate MDOR performance in older

155 healthy participants, and to compare it to that in younger participants.

156

157 Methods

158 Ethics and Participants

159 A consecutive, convenience sample of healthy, older, adult participants, with normal or 160 corrected to normal vision was recruited from the local community, under ethical approval

161 from the University of Liverpool Research Ethics committee (Reference No. 2933). We did not

162 conduct a formal power calculation. All participants provided informed, written consent after

163 the experiment was explained to them and they had an opportunity to ask questions.

164 Participants were offered $\mathrm{f} 10$ to compensate them for their time and for the expense of

165 travelling in to the University for testing. Comparison data were available from younger

166 participants from previous experiments. We identified participants aged between 18y and 28y

167 tested previously on the same MDOR task, using the same equipment and procedures.

Apparatus and Stimuli 
169 We used the same apparatus and stimuli as in earlier experiments (Knox et al. 2018; Wolohan \& 170 Knox 2014). Briefly, stimuli were presented on a 21" monitor (1024×768 spatial resolution, 100

$171 \mathrm{~Hz}$ temporal resolution) driven by a VSG2/5 card (Cambridge Research Systems, Rochester, UK), 172 positioned on the fronto-parallel plane $57 \mathrm{~cm}$ from the participant's eye. Horizontal eye 173 position of the left eye was recorded using a Skalar Iris IR Eye Tracker, with the eye tracker 174 output digitised with 16-bit precision using a CED Power 1401 (Cambridge Electronic Design, 175 Cambridge, UK) interface. Oculomotor data was stored for off-line, trial-by-trial analysis using 176 custom software.

177 We used the synchronous version of the MDOR task (Knox et al, 2018; Fig 1) in which a central 178 fixation target $\left(0.2^{\circ}\right.$ black square; $\left.11 \mathrm{~cd} / \mathrm{m}^{2}\right)$ was presented on a light background $\left(56 \mathrm{~cd} / \mathrm{m}^{2}\right.$; contrast 79\%) for a randomised period of $0.5-1.5 \mathrm{~s}$. Immediately when it was extinguished, the saccade target (a $0.2^{\circ}$ black square) appeared $5^{\circ}$ to the left or to the right of fixation (randomised and with equal frequency) and was displayed for either 200ms or 1000ms (randomised from trial to trial). Participants were instructed to maintain fixation in the centre of the display until the saccade target disappeared, when they were to execute a saccade to the target's location (i.e. saccade on target offset), pause, and return their gaze to the centre of the display in preparation for the next trial. They were explicitly instructed not to saccade to the onset of the target. Before testing commenced, the trial sequence was demonstrated to participants; this was repeated until they were happy that they understood what was required.

\section{Procedures}

Participants were carefully positioned by adjusting table height, a chin rest and cheek pads. They completed two runs of 120 MDOR trials usually with a break in between them. The quality of performance was carefully monitored to ensure that it was maintained, with verbal feedback given as necessary. A 32-trial calibration procedure was performed after each run. In calibration trials, after a randomised fixation period (0.5-1.5s), the fixation target was extinguished and a saccade target was presented to the left or right at an eccentricity of $5^{\circ}$ or $10^{\circ}$ (randomised and with equal frequency) for 1 s. Participants were instructed to fixate the central point and saccade to the target as soon as it appeared, fixating it until it was extinguished, at which point 
197 they could blink and return to the centre, ready for the next trial. These procedures were

198 identical to those used in earlier experiments. Older participants also completed the

199 Addenbrookes Cognitive Examination (ACE) III questionnaire (Hsieh et al. 2013).

\section{Analysis}

201 Oculomotor data were analysed using an interactive program which displayed the eye position

202

203

204

205

206

207

208

209

210

211

212

213

214

215

216

217

218

219

220

221

222

223 data and the time at which the "go" signal (target offset in MDOR trials) occurred. The calibration data were used to transform the data from arbitrary system units into units of degrees of eye rotation, allowing us to measure saccade amplitude. Trials with blinks or unstable fixation prior to target appearance were removed from the analysis. For each valid trial (ie trials free of blinks and poor fixation), the latency and amplitude of the primary targetdirected saccade were measured.

Data were collated in MS Excel. Error responses were first identified, removed and collated separately from correct trials, and the error rate calculated. Any target directed saccade with an amplitude greater than $1^{\circ}$ that occurred from $80 \mathrm{~ms}$ after target onset to $80 \mathrm{~ms}$ after target offset was counted as an error. Saccades occurring $<80 \mathrm{~ms}$ after target onset were classified as anticipatory saccades, in common with many other studies (Amatya et al. 2011, Wolohan \& Knox 2014), and not included in further analysis. Any target directed saccade occurring from $80 \mathrm{~ms}$ to $1000 \mathrm{~ms}$ after target offset was counted as a correct response. Note that in comparison data for younger participants taken from a previously published study (Knox et al. 2018), this period was $80 \mathrm{~ms}$ to $600 \mathrm{~ms}$ after target offset. In the current data, this additional $400 \mathrm{~ms}$ period accounted on average for $2.5 \%$ of correct responses.

For each participant, median saccade latency was calculated along with the error rate. Across participants, both latency and error rate were summarised using the mean. Error and latency data for the two target display durations (200ms and $1000 \mathrm{~ms})$ were kept separate. We used the median saccade latency from calibration data, collapsed across direction and eccentricity, to provide an estimate of reflexive prosaccade latency, rather than use control MDOR runs. This reduced the testing time for older participants, and similar (calibration) data were available for 
224 younger participants tested previously. Statistical analysis (details in Results) was conducted 225 with SPSS v22.

226

\section{Results}

228 Twenty-two older participants (13 male) were recruited and tested in the present study. The mean $( \pm S D)$ age of this group was $62 \pm 7 y$ (range: $50 y$ to $72 y$ ). Eight participants were aged 50y$59 y$, eight 60y-69y and six 70y and over. The mean ACE III total score for these older participants was 96 \pm 3 (range 91-100). Figure 2 illustrates both individual and group latency and error rate data for these participants. Across the older group the mean number of trials available for analysis per participant was 200 . The mean proportion of valid trials contributing to the analysis was $83 \%$ (ie $17 \%$ of trials were lost to blinks, poor fixation, or had no responses). As in previous experiments with the MDOR task, latency was longer than would be expected for prosaccade responses, and modulated by the target display duration (TDD). In tasks with a $200 \mathrm{~ms}$ TDD, mean group latency was $462 \pm 103$ ms compared to latency in the calibration task of $236 \pm 57 \mathrm{~ms}$. For the $1000 \mathrm{~ms}$ TDD, mean latency was $332 \pm 80 \mathrm{~ms}$. Similarly, for error rate we observed the same general pattern as in previous experiments; for a TDD of 200ms the mean error rate was $18 \pm 18 \%$ and for a TDD of 1000 ms it was $46 \pm 23 \%$.

MDOR task performance in the older group was compared to that in younger participants tested previously (Knox et al. 2018; Wolohan \& Knox 2014). We used data from a group of 39 younger participants, with a group mean age of 22 2 y (range 19y-27y; 15 male). The mean number of trials available per participant was 187 (slightly lower than for the older group), and the mean proportion of trials analysed per participant was $78 \%$ (ie a rejection rate of $22 \%$ ). The difference in the per-participant proportion of trials available for analysis between the two groups was not statistically significant $(t=2.0 ; d f=54 ; p=0.22)$. When compared to this younger group, there was an apparent ageing effect on raw latency (Fig. 3a). For the 200ms condition the latencies were $462 \pm 103 \mathrm{~ms}$ and $394 \pm 61 \mathrm{~ms}$ in old and young groups respectively (a 
252 difference of $68 \mathrm{~ms}$ ) while for the $1000 \mathrm{~ms}$ condition they were $332 \pm 80 \mathrm{~ms}$ and $298 \pm 46 \mathrm{~ms}$ (a

253 difference of $34 \mathrm{~ms}$ ). When tested with a repeated measures ANOVA with group (old, young) as

254 a between and TDD (200ms, $1000 \mathrm{~ms})$ as a within-subjects factor, both factors returned

255 statistically significant results (group: $F_{1,59}=14.1, p<0.001, \eta_{p}{ }^{2}=0.19 ;$ TDD: $F_{1,59}=78.0, p<0.001$,

$\left.256 \eta_{p}^{2}=0.57\right)$ with no statistically significant interaction $\left(F_{1,59}=1.73, p=0.19\right)$.

257

258

We also calculated effect size using Cohen's $d$ (sometimes called $d_{s}$ as the sample rather than

259 population means and standard deviations are used, with Bessel's correction; Lakens 2013). For each participant we calculated the mean of the latencies for the two TDDs, and then calculated the group mean and standard deviation (old: $397 \pm 55 \mathrm{~ms}$; young: $346 \pm 49 \mathrm{~ms}$ ). Cohen's $d$ was 0.99 . Given the relatively small sample size, and that this can artificially inflate $d$, we also calculated the corrected effect size (Hedge's $g_{s}$ ). However, the correction factor was only 0.000088 (ie d was left essentially unchanged).

There was a significant difference in latency between old $(236 \pm 57 \mathrm{~ms})$ and young $(189 \pm 41 \mathrm{~ms})$ groups for reflexive prosaccade latency, which was available from the calibration task ( $\mathrm{t}=3.4$; $d f=33 ; p=0.002 ; d=0.92$ ). For each participant we modified the MDOR latency by subtracting their calibration latency, to take account of general age-related changes in the saccade circuitry. This abolished the latency difference between groups (Fig. 3b).

Given that the group data suggested the modulation of latency by TDD might differ between the two groups (although the interaction term in the ANOVA was not statistically significant), we calculated the difference in latency between the $200 \mathrm{~ms}$ and $1000 \mathrm{~ms}$ conditions for each participant and then summarised for the groups. The mean difference was $164 \pm 81 \mathrm{~ms}$ in the older group and $96 \pm 47 \mathrm{~ms}$ in the younger group, statistically significantly different $(t=3.4 ; \mathrm{df}=26$; $p=0.002 ; d=1.12$ ). To account for the generally longer latencies in the older participants, we also calculated this difference as a percentage of the average latency of the two TDDs. This yielded a difference of $40 \pm 15 \%$ for the older and $28 \pm 12 \%$ for the younger group; these differences remained statistically significant $(t=3.2 ; d f=33 ; p=0.003 ; d=0.92)$. The data of two older 
281 participants with very high error rates and therefore very few correct trials (who did not exhibit

282 the usual pattern of higher latency in the $200 \mathrm{~ms}$ condition) were removed from this analysis.

MDOR error rates were higher in the older group compared to younger participants (200ms condition: $18 \pm 18 \%$ vs $10 \pm 8 \%$; 1000 ms condition: $46 \pm 23 \%$ vs $28 \pm 14 \%$; Fig 4$)$. Using a repeated measures ANOVA similar to that described above (group as a between and TDD as a within subjects factor) both group $\left(F_{1,59}=12.6, p=0.001, \eta_{p}{ }^{2}=0.18\right)$ and TDD $\left(F_{1,59}=200.7, p<0.001\right.$, $\eta_{p}{ }^{2}=0.77$ ) returned statistically significant results. In contrast to the results with raw latency, the interaction between group and TDD was also statistically significant $\left(F_{1,59}=7.4, p=0.009\right.$, $\left.\eta_{p}{ }^{2}=0.11\right)$. Investigating the group difference for the two TDD's further using post-hoc t-tests and the sequential Holm-Sidak procedure to control for Type I errors, we found that the difference in error rate for the $200 \mathrm{~ms}$ condition did not reach statistical significance $(t=2.19, \mathrm{df}=$ 26; corrected $p=0.073$ ) while the error rate for the $1000 \mathrm{~ms}$ did $(t=3.31, d f=30$, corrected $p=0.002)$. Cohen's $d$ for the error rate was 1.01 .

295

The distribution of both error and correct response timings was explored by plotting the pooled distributions for both old and young groups of participants for both target display durations (Fig. 5). For both groups, there were clear peaks after target onset in a latency range consistent with them being failed attempts to inhibit the response to target onset (time=0ms; indicated by the vertical dotted lines in Fig. 5). These peaks occurred in the same latency bins (bin width $=50 \mathrm{~ms}$ ) for both old and young participants (the $-50 \mathrm{~ms}$ bin for the $200 \mathrm{~ms}$ condition, Fig. $5 a, c$; the $-850 m s$ bin for the $1000 m s$ condition, Fig $5 b, d)$. For both conditions, these bins are the fourth bin after target appearance, comprising responses with latencies in the rage 150-199ms. We defined latency ranges which captured these early peaks (for the 200ms condition: -100ms to $150 \mathrm{~ms}$; for $1000 \mathrm{~ms}:-950 \mathrm{~ms}$ to $-650 \mathrm{~ms}$; Fig. 5) and recalculated error rates to include only responses in these ranges. This had relatively little effect on error rates for the $200 \mathrm{~ms}$ condition (old: $20 \pm 18 \%$; young: $11 \pm 8 \%$ ), and a more marked effect on error rates in the $1000 \mathrm{~ms}$ condition (old:26 $\pm 20 \%$; young: $15 \pm 12 \%$ ). We retested these data with the same design of ANOVA as above; group $\left(F_{1,59}=8.1, p=0.006, \eta_{p}{ }^{2}=0.121\right)$ and TDD $\left(F_{1,59}=12.3, p=0.001, \eta_{p}^{2}\right.$ 
$310=0.121)$ returned statistically significant results, while the interaction was no longer significant

$311\left(F_{1,59}=0.7, p=0.41\right)$. We computed the effect size (" $d$ ") for TDD's of 200ms and 1000ms

312 separately and these were 0.67 and 0.72 respectively. For the $1000 \mathrm{~ms}$ condition we also

313 examined the residual error rate defined as the proportion of responses occurring from $-600 \mathrm{~ms}$

314 to $0 \mathrm{~ms}$. This was again higher for the older group ( $21 \pm 10 \%$ vs $12 \pm 7 \% ; t=3.6, d f=35 ; p=0.001 ; d=$

315 1.02).

316

317 Discussion

318 Studies of oculomotor control have been widely used over a long period to investigate the 319 effects of ageing (Abel et al. 1983; Morrow \& Sharpe 1993; Olincy et al. 1997; Simons \& Buttner 320 1985; Spooner et al. 1980). In general, as might be expected, oculomotor performance declines 321 with age. The effect of ageing on antisaccade (AS) performance has also been widely studied, 322 with there being general agreement that AS latency and error rate both increase with age 323 (Butler et al. 1999; Olincy et al. 1997). AS task performance is often taken to provide an index of inhibitory control, and therefore its decline with age is assumed to support the hypothesis of a general age-related deterioration in inhibitory function (Hasher \& Zacks 1988). However, while clearly involving inhibitory control, the AS task involves other key aspects of executive function, and AS directional error rate is influenced by multiple factors (Bowling et al. 2012; Lee et al. 2010; Magnusdottir et al. 2019). Our motivation in developing the MDOR task was to target oculomotor inhibitory control more precisely, and in the present study to investigate the effects of normal ageing on MDOR performance.

We have discussed previously the differences between the MDOR task and other types of saccade tasks, such as the memory guided saccade task (MGS) which it resembles (Knox et al, 2018). In the MDOR task there is no extended memory delay (typically a delay of several seconds in the MGS task), the target is present throughout the fixation period and the go signal is provided by a transient at the target position, not fixation. The MDOR task also resembles other delayed saccade tasks, which lack the working memory load of MGS tasks (Hutton et al. 2002; Reuter et al. 2007). However, there are important differences in detail in between 
339

340

341

342

343

344

345

346

347

348

349

350

351

352

353

354

355

356

357

358

359

360

361

362

363

364

365

366

367

studies relating to the timing of the removal of the central fixation target, and the nature and timing of the go signal (eg an auditory tone was used in Reuter et al. 2007, not a target offset).

Such methodological differences should be borne in mind when comparing studies as they are likely to affect both error rate and latency.

We have also discussed previously other effects which might contribute to the pattern of results observed in the MDOR task (Knox et al. 2018; Wolohan \& Knox 2013). The magnitude of the latency increases we have observed are much larger than can be accounted for by preview effects, or the lower salience of target offsets compared to target onsets. It is in tasks in which oculomotor responses have to be inhibited that latency increases of this magnitude are observed (Machado \& Rafal 2000).

More generally two broad types or classes of inhibitory control have been defined. Proactive inhibitory control involves top-down, preparatory inhibition before responses are initiated, whereas reactive inhibitory control involves the inhibition of an initiated or prepotent action, often in response to a cue or signal (Aron 2011; Verbruggen et al. 2014b). The classic antisaccade task (in which blocks of only antisaccade are run) contains both proactive and reactive aspects, a complication noted by Aron (2011) in his classification of tasks (eg see his Table 1). The MDOR task is perhaps more proactive in nature in that in every trial in a run or block the emphasis is on the participant not executing a saccade to the target when it appears. Nevertheless, there is still a reactive element in that, as with the antisaccade task, the appearance of the target (the external "cue") triggers saccade programming in each trial, and it is this that must be inhibited.

We recruited a group of healthy (by self-report) older participants and screened them with the ACE III questionnaire. All scored above the cut-off for suspicion of neurological disease (usually quoted as a total score of 88) and the group mean was very similar to that reported in Hsieh et al. (2013) for a similarly aged control group. MDOR performance in our older participants was qualitatively similar to that observed previously (Knox et al. 2018; Wolohan \& Knox 2014). 
368

369

370

371

372

373

374

375

376

377

378

379

380

381

382

383

Latency in correct trials was much longer than is consistent with reflexive prosaccades, and was modulated by target display duration (TDD). We did not run a control MDOR task in the current experiment as we have done previously, in part to reduce the time commitment demanded of our participants and to avoid participant fatigue. However, the calibration task that was run, which is identical to that which we have used in previous studies, is a prosaccade task, with randomised target direction and randomised fixation duration. It therefore provides a means of obtaining an estimate for prosaccade latency in our participants. Comparing MDOR latency with that observed in the calibration task, latency was increased by $226 \mathrm{~ms}$ in the $200 \mathrm{~ms}$ TDD condition over calibration task latency, and by $96 \mathrm{~ms}$ in the $1000 \mathrm{~ms}$ condition; the magnitude of these increases is similar to those reported when the comparison is with an MDOR control task (see Knox et al. 2018:Table 1).

Latency was increased in the older compared to younger participants, by an average of 68ms in the $200 \mathrm{~ms}$ condition and $34 \mathrm{~ms}$ in the $1000 \mathrm{~ms}$ condition. These are of an order that might be expected given ageing effects on latency in other saccade tasks. What is of more interest is the extent to which these differences are specific to the MDOR task (and therefore indicative of changes in inhibitory control in ageing), or simply a reflection of a general slowing in the oculomotor system (Verhaeghen 2011). For prosaccade tasks, latency increases with age, although the reported increases are variable, dependant on factors such as the exact age of participant groups, and the type of task used. The difference of $47 \mathrm{~ms}$ observed between our young and old groups on the calibration prosaccade task is similar to that reported elsewhere for similarly aged groups (Eenshuistra et al. 2004; Klein et al. 2000).

When we corrected MDOR latency for both groups by subtracting prosaccade latencies from MDOR latencies, the latency difference between groups was abolished, suggesting that most if not all of the latency increase in the MDOR task could be due to general slowing in the saccade system in older participants. A similar result has been reported for antisaccades (Butler et al. 1999). Given the differences between the calibration and MDOR tasks, this result should be 
396

397

398

399

400

401

402

403

404

405

406

407

408

409

410

411

412

413

414

415

416

417

418

419

420

421

422

423

424

treated with caution. The calibration task was not simply a repetition of the MDOR task without inhibition, as is the MDOR control task.

Increased latency in the MDOR task is indicative of the requirement to inhibit the reflexive response to target onset (discussed in detail in Knox et al. 2018). The modulation in latency with TDD is consistent with higher levels of inhibition if the target offset (the go signal) occurs soon after target onset (as in the $200 \mathrm{~ms}$ condition), with the level of inhibition declining the longer the target is displayed. In pilot studies we have observed a monotonic relationship between latency and TDD tested over a wide range (Knox \& Abd Razak 2010). The larger latency modulation in the older group, which was still present once the generally longer latency in the older group was accounted for, suggests either a higher level of inhibition initially, a lower level of inhibition eventually, or a combination of both, compared to the younger group. This could be compatible with the older participants exerting a great inhibitory effort as a strategy for improving performance overall, with the implication that this cannot be maintained as effectively as in the younger participants, leading to a larger difference in latency between the two TDD conditions used here.

Higher MDOR error rates in the older group relative to the younger group parallels a similar finding in the AS task (Abel \& Douglas 2007; Klein et al. 2000; Peltsch et al. 2011). We have shown previously that although both the AS and MDOR tasks involve the inhibition of reflexive prosaccades, the error rates in the two tasks do not correlate when compared in the same participants (Wolohan \& Knox 2014). On this basis, closely comparing error rates between the two task types is probably of little value. However, while they may not correlate it is worth noting that in general error rates in these two tasks are similar, and that interparticipant variability in older participants tends also to be increased for both tasks.

While none of our participants reached a $100 \%$ error rate in the current study, one older participant reached $96 \%$ in the 1000 ms condition. As with the AS task, precise error rates are influenced by a variety of factors including the details of task design, numbers of trials run, 
425 block structure and so on, many of which are yet to be investigated for the MDOR task. But

426 bearing in mind that one use of the MDOR task might be in patient populations, in which higher

427 rates might be anticipated compared to the healthy participants studied here, using a version

428 with generally lower error rates, and reduced variability, would be an advantage. Both error

429 rate and latency in the AS task are modified by changing fixation conditions. In many clinical AS

430 studies this means that a gap AS task is used to avoid floor effects (i.e. 0\% error rates) in control

431 groups. However, this option is not available with the MDOR task (Knox et al. 2018). But

432 investigating other stimulus and task features (e.g. the provision of more spatial information by

433 using placeholders, the use of three TDDs as opposed to two) might generally improve

434 performance without fundamentally altering the ability of the task to provide insights into

435 inhibitory control.

436

437 The error rates that we calculated captured all errors (defined as responses occurring between $>80 \mathrm{~ms}$ post target onset and $<80 \mathrm{~ms}$ after target offset) as a proportion of the total number of valid responses. However, the average distributions (Fig. 5) suggest that only some of these errors are uninhibited responses to the target onset. These are the responses that make up the early peaks, around the $-50 \mathrm{~ms} /-850 \mathrm{~ms}$ bins in Fig. 5 . Recalculating the error rate to capture only these early responses perhaps provides a purer measurement of behavioural inhibition in the MDOR task. On this basis, error rate remained significantly higher in older participants, implying weaker inhibitory control. This measure might have the advantage of avoiding ceiling effects in participants with known problems with inhibition, particularly for longer TDDs. However, this does raise the issue of how to classify the other errors which occurred at a low rate throughout the fixation period in the $1000 \mathrm{~ms}$ condition. This "residual error rate" was also significantly higher in the older participants. Conceptually it seems plausible that the residual error rate represents a different aspect of inhibitory function. While it is possible that these errors are also uninhibited responses to target onsets, those occurring at long latencies might reflect the ability of participants to maintain fixation (i.e. maintain inhibition) in circumstances where a particular action is anticipated. In this context it is worth noting that neither in the 
454

455

456

457

458

459

460

461

462

463

464

465

466

467

468

469

470

471

472

473

474

475

476

477

478

479

480

481

482

on the fixation period in the $1000 \mathrm{~ms}$ condition that might represent target offset anticipations.

This suggests that with only two TDDs (and randomisation of direction) there is sufficient uncertainty in the task to prevent participants successfully anticipating the target offset.

Given the clear difference between groups in error rates, it appears that even if older participants adopt a different strategy by, for example, exerting a maximal inhibitory effort at target onset (which they find difficult to maintain at long TDDs), reflected in increased latency, they still cannot inhibit their oculomotor responses as successfully as younger participants. How this relates to other aspects of inhibition remains to be seen. While a wide range of tasks have been used to investigate inhibition, correlations between them are absent or low (Friedman \& Miyake 2004; Rey-Mermet et al. 2018). This is often taken to be due to the general problem of task impurity (which we have also argued is a problem with the AS task). The MDOR task is clearly not immune to exactly the same problem. There is a working memory load in the MDOR task (e.g. there is an instruction to be remembered). However, it is reduced compared to the AS task. Nor would it be true to say that there is no attentional load in the MDOR task. But as the target position for the response is the same as the position of the target onset, there is no requirement to disengage, shift and re-engage spatial attention at a non-target location, as there is in the AS task. So the observed effects are more closely tied to the ability of participants to inhibit their responses.

\section{Conclusion}

The MDOR task provides a useful means of studying oculomotor inhibition. Latency increases in the MDOR task with age appear to primarily reflect general age-related slowing of the saccade system. However, the higher MDOR error rates observed in the older group, particularly for those errors consistent with uninhibited responses to target onsets, confirms an inhibitory control deficit in normally ageing participants.

\section{Acknowledgements}

We are grateful to all the participants who took part in these experiments. 
483 
485

486

487

488

489

490

491

492

493

494

495

496

497

498

499

500

501

502

503

504

505

506

507

508

509

510

511

512

513

514

515

516

517

518

519

520

521

522

523

524

525

526

527

528

529

530

531

\section{References}

Abel LA, and Douglas J. 2007. Effects of age on latency and error generation in internally mediated saccades. Neurobiology of Aging 28:627-637. https://doi.org/10.1016/j.neurobiolaging.2006.02.003

Abel LA, Troost BT, and Dell'Osso LF. 1983. The effects of age on normal saccadic characteristics and their variability. Vision Res 23:33-37.

Alichniewicz KK, Brunner F, Klünemann HH, and Greenlee MW. 2013. Neural correlates of saccadic inhibition in healthy elderly and patients with amnestic mild cognitive impairment. Frontiers in Psychology 4. 10.3389/fpsyg.2013.00467

Amatya N, Gong Q, and Knox PC. 2011. Differing proportions of 'express saccade makers' in different human populations. Experimental Brain Research 210:117-129. 10.1007/s00221-011-2609-z

Aron AR. 2011. From reactive to proactive and selective control: Developing a richer model for stopping inappropriate responses. Biological Psychiatry 69:e55-e68. http://dx.doi.org/10.1016/j.biopsych.2010.07.024

Baird-Gunning J, and Lueck C. 2018. Central control of eye movements. Current Opinion in Neurology 31:90-95. 10.1097/WCO.0000000000000514

Bowling AC, Hindman EA, and Donnelly JF. 2012. Prosaccade errors in the antisaccade task: differences between corrected and uncorrected errors and links to neuropsychological tests. Experimental Brain Research 216:169-179. 10.1007/s00221-011-2921-7

Butler KM, Zacks RT, and Henderson JM. 1999. Suppression of reflexive saccades in younger and older adults: Age comparisons on an antisaccade task. Memory \& Cognition 27:584-591. $10.3758 / \mathrm{bf03211552}$

Castiglione A, Wagner J, Anderson M, and Aron AR. 2019. Preventing a thought from coming to mind elicits increased right frontal beta just as stopping action does. Cerebral Cortex 29:2160-2172. 10.1093/cercor/bhz017

Crawford T, Parker E, Solis-Trapala I, and Mayes J. 2011. Is the relationship of prosaccade reaction times and antisaccade errors mediated by working memory? Experimental Brain Research 208:385397. 10.1007/s00221-010-2488-8

Crawford TJ, Bennett D, Lekwuwa G, Shaunak S, and Deakin JFW. 2002. Cognition and the inhibitory control of saccades in schizophrenia and Parkinson's disease. Progress in Brain Research 140:449-466.

Crawford TJ, Higham S, Renvoize T, Patel J, Dale M, Suriya A, and Tetley S. 2005. Inhibitory control of saccadic eye movements and cognitive impairment in Alzheimer's disease. Biological Psychiatry 57:1052-1060. http://dx.doi.org/10.1016/j.biopsych.2005.01.017

Diarra M, Zendel BR, Benady-Chorney J, Blanchette C-A, Lepore F, Peretz I, Belleville S, and West GL. 2019. Playing Super Mario increases oculomotor inhibition and frontal eye field grey matter in older adults. Experimental Brain Research 237:723-733. 10.1007/s00221-018-5453-6

Donders FC. 1969. On the speed of mental processes. Acta Psychologica 30:412-431.

Eenshuistra RM, Ridderinkhof KR, and Molen MWvd. 2004. Age-related changes in antisaccade task performance: Inhibitory control or working-memory engagement? Brain and Cognition 56:177188. https://doi.org/10.1016/j.bandc.2004.02.077

Fernandez-Ruiz J, Peltsch A, Alahyane N, Brien DC, Coe BC, Garcia A, and Munoz DP. 2018. Age related prefrontal compensatory mechanisms for inhibitory control in the antisaccade task. Neurolmage 165:92-101. https://doi.org/10.1016/i.neuroimage.2017.10.001

Friedman NP, and Miyake A. 2004. The relations among inhibition and interference control functions: A latent-variable analysis. Journal of Experimental Psychology: General 133:101-135. 10.1037/0096-3445.133.1.101

Peer) reviewing PDF | (2019:05:37620:3:0:NEW 4 Dec 2019) 
532

Gaspelin N, and Luck SJ. 2018. The role of inhibition in avoiding distraction by salient stimuli. Trends in Cognitive Sciences 22:79-92. https://doi.org/10.1016/j.tics.2017.11.001

Hallett P. 1978. Primary and secondary saccades to goals defined by instructions. Vision Research 18:1279-1296.

Hanes DP, and Carpenter RHS. 1999. Countermanding saccades in humans. Vision Research 39:27772791.

Hasher L, and Zacks R. 1988. Working memory, comprehension, and aging: A review and a new view. In: Bower GH, ed. The Psychology of Learning and Motivation. Sna Diego, CA: Academic Press, 193225.

Hsieh S, Schubert S, Hoon C, Mioshi E, and Hodges JR. 2013. Validation of the Addenbrooke's Cognitive Examination III in frontotemporal dementia and Alzheimer's disease. Dementia and Geriatric Cognitive Disorders 36:242-250. 10.1159/000351671

Hutton SB, and Ettinger U. 2006. The antisaccade task as a research tool in psychopathology: A critical review. Psychophysiology 43:302-313.

Hutton SB, Joyce EM, Barnes TRE, and Kennard C. 2002. Saccadic distractibility in first-episode schizophrenia. Neuropsycholgia 40:1729-1726.

Kaufman LD, Pratt J, Levine B, and Black SE. 2012. Executive deficits detected in mild Alzheimer's disease using the antisaccade task. Brain and Behavior 2:15-21. 10.1002/brb3.28

Klein C, Fischer B, Hartnegg K, Heiss W, and M. R. 2000. Optomotor and neuropsychological performance in old age. Exp Brain Res 135:141-154.

Knox PC, and Abd Razak N. 2010. Oculomotor inhibition: Shared or separate mechanisms for saccades and smooth pursuit? Soc Neuroscience Absts 676:17.

Knox PC, Heming De-Allie E, and Wolohan FDA. 2018. Probing oculomotor inhibition with the minimally delayed oculomotor response task. Experimental Brain Research 236:2867-2876. 10.1007/s00221-018-5345-9

Lakens D. 2013. Calculating and reporting effect sizes to facilitate cumulative science: a practical primer for t-tests and ANOVAs. Frontiers in Psychology 4. 10.3389/fpsyg.2013.00863

Lee H, Abegg M, Rodriguez A, Koehn J, and Barton JJS. 2010. Why do humans make antisaccade errors? Experimental Brain Research 201:65-73. 10.1007/s00221-009-2008-x

Lennertz L, Rampacher F, Vogeley A, Schulze-Rauschenbach S, Pukrop R, Ruhrmann S, Klosterkötter J, Maier W, Falkai P, and Wagner M. 2012. Antisaccade performance in patients with obsessivecompulsive disorder and unaffected relatives: further evidence for impaired response inhibition as a candidate endophenotype. European Archives of Psychiatry and Clinical Neuroscience 262:625-634. 10.1007/s00406-012-0311-1

Logan GD, and Cowan WB. 1984. On the ability to inhibit thought and action -a theory of an act of control. Psychological Review 91:295-327. 10.1037/0033-295x.91.3.295

Machado L, and Rafal R. 2000. Control of eye movement reflexes. Exp Brain Res 135:73-80.

Magnusdottir BB, Faiola E, Harms C, Sigurdsson E, Ettinger U, and Haraldsson HM. 2019. Cognitive measures and performance on the antisaccade eye movement task. Journal of Cognition 2:1-13.

Mirsky JB, Heuer HW, Jafari A, Kramer JH, Schenk AK, Viskontas IV, Miller BL, and Boxer AL. 2011. Antisaccade performance predicts executive function and brain structure in normal elders. Cognitive and behavioral neurology : official journal of the Society for Behavioral and Cognitive Neurology 24:50-58. 10.1097/WNN.0b013e318223f6c6

Miyake A, and Friedman NP. 2012. The nature and organization of individual differences in executive functions: Four general conclusions. Current Directions in Psychological Science 21:8-14. $10.1177 / 0963721411429458$ 
578

579

580

581

582

583

584

585

586

587

588

589

590

591

592

593

594

595

596

597

598

599

600

601

602

603

604

605

606

607

608

609

610

611

612

613

614

615

616

617

618

619

620

621

622

623

624
Miyake A, Friedman NP, Emerson MJ, Witzki AH, Howerter A, and Wager TD. 2000. The unity and diversity of executive functions and their contributions to complex "frontal lobe" tasks: A latent variable analysis. Cognitive Psychology 41:49-100. http://dx.doi.org/10.1006/cogp.1999.0734

Morrow MJ, and Sharpe JA. 1993. Smooth pursuit initiation in young and elderly subjects. Vision Res 33:203-210.

Munoz DP, Broughton JR, Goldring JE, and Armstrong IT. 1998. Age-related performance of human subjects on saccadic eye movement tasks. Experimental Brain Research 121:391-400.

Munoz DP, and Everling S. 2004. Look away: the anti-saccade task and the voluntary control of eye movement. Nat Rev Neurosci 5:218-228.

Noorani I, and Carpenter RHS. 2013. Antisaccades as decisions: LATER model predicts latency distributions and error responses. European Journal of Neuroscience 37:330-338. 10.1111/ejn.12025

Olincy A, Ross R, Young D, and Freedman R. 1997. Age diminishes performance on an antisaccade eye movement task. Neurobiol Aging 18:483-489.

Pa J, Dutt S, Mirsky JB, Heuer HW, Keselman P, Kong E, Trujillo A, Gazzaley A, Kramer JH, Seeley WW, Miller BL, and Boxer AL. 2014. The functional oculomotor network and saccadic cognitive control in healthy elders. Neurolmage 95:61-68. http://dx.doi.org/10.1016/j.neuroimage.2014.03.051

Peltsch A, Hemraj A, Garcia A, and Munoz DP. 2011. Age-related trends in saccade characteristics among the elderly. Neurobiology of Aging 32:669-679. http://dx.doi.org/10.1016/j.neurobiolaging.2009.04.001

Reuter B, Jäger M, Bottlender R, and Kathmann N. 2007. Impaired action control in schizophrenia: The role of volitional saccade initiation. Neuropsychologia 45:1840-1848.

http://dx.doi.org/10.1016/j.neuropsychologia.2006.12.006

Reuter B, Rakusan L, and Kathmanna N. 2005. Poor antisaccade performance in schizophrenia: An inhibition deficit? Psychiatry Research 135:1-10. http://dx.doi.org/10.1016/j.psychres.2004.12.006

Rey-Mermet A, and Gade M. 2017. Inhibition in aging: What is preserved? What declines? A metaanalysis. Psychonomic Bulletin \& Review. 10.3758/s13423-017-1384-7

Rey-Mermet A, Gade M, and Oberauer K. 2018. Should we stop thinking about inhibition? Searching for individual and age differences in inhibition ability. Journal of Experimental Psychology: Learning, Memory, and Cognition 44:501-526. 10.1037/xIm000045010.1037/xlm0000450.supp (Supplemental)

Salthouse T. 2012. Consequences of age-related cognitive declines. Annual Review of Psychology 63:201226. 10.1146/annurev-psych-120710-100328

Simons B, and Buttner U. 1985. The influence of age on OKN. Eur Arch Psychiatr Neurol Sci 234:369-373. Spooner JW, Sakala SM, and Baloh RW. 1980. Effect of ageing on eye tracking. Arch Neurol 37:575-576.

Verbruggen F, Best M, Bowditch WA, Stevens T, and McLaren IPL. 2014. The inhibitory control reflex. Neuropsychologia 65:263-278. https://doi.org/10.1016/j.neuropsychologia.2014.08.014

Verbruggen F, and Logan GD. 2008. Response inhibition in the stop-signal paradigm. Trends in Cognitive Sciences 12:418-424. https://doi.org/10.1016/j.tics.2008.07.005

Verhaeghen P. 2011. Aging and executive control: Reports of a demise greatly exaggerated. Current Directions in Psychological Science 20:174-180. 10.1177/0963721411408772

Verhaeghen P. 2014. Age-related differences in the speed of executive control. The Elements of Cognitive Aging: Meta-Analyses of Age-Related Differences in Processing Speed and Their Consequences Oxford University Press; Oxford Scholarship Online, 1-63.

Wolohan F, and Knox P. 2013. Examining oculomotor inhibition in express saccade makers. Society for Neuroscience Online 364:16.

Peer] reviewing PDF | (2019:05:37620:3:0:NEW 4 Dec 2019) 
625 Wolohan FDA, and Knox PC. 2014. Oculomotor inhibitory control in express saccade makers.

626 Experimental Brain Research 232:3949-3963. 10.1007/s00221-014-4076-9

627 
Figure 1

Description of the MDOR task.

a. Trial structure. After a randomised fixation period of $0.5 \mathrm{~s}$ to $1.5 \mathrm{~s}$, the target appeared 5 degrees to either the left or right. Participants were instructed to look to the target position when it disappeared (i.e. saccade on offset). b. Trial and response timings. Targets were displayed for either $200 \mathrm{~ms}$ or $1000 \mathrm{~ms}$. The example correct saccade, executed in response to a target displayed for $1000 \mathrm{~ms}$, occurs after the offset. The example error saccade occurs soon after the onset, well before either of the possible offsets.

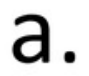

Fixation: $0.5-1.5 \mathrm{~s}$

.

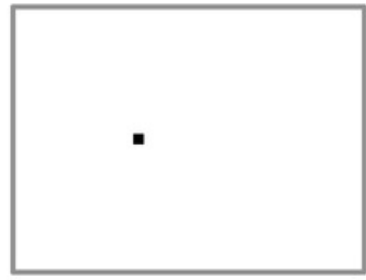

Target onset Display duration $=200 \mathrm{~ms}$ or $1000 \mathrm{~ms}$ Direction randomised

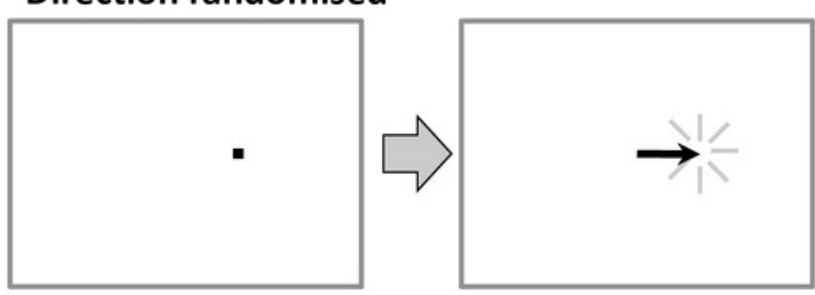

b.

Task instruction: 'Saccade to the target offset'

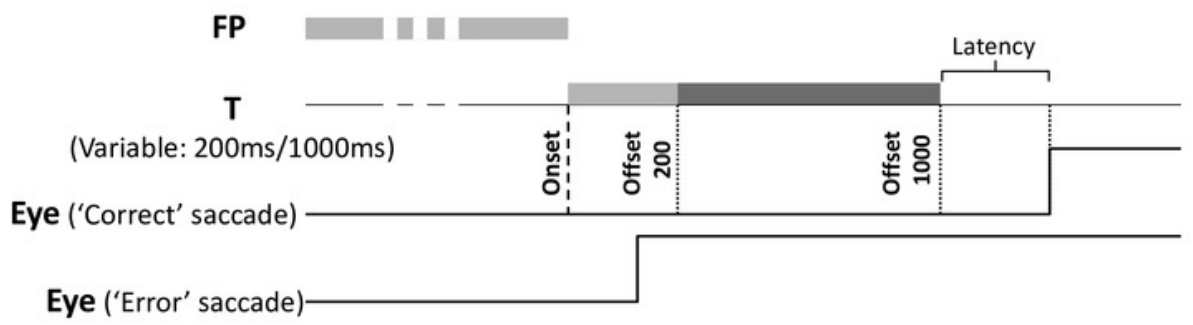


Figure 2

MDOR latency and error rate in older participants.

(A) Latency, (B) Error Rate, observed in 22 older participants. Grey data points show individual results; horizontal bars show group mean $\pm 95 \% \mathrm{Cl}$.

a. Latency
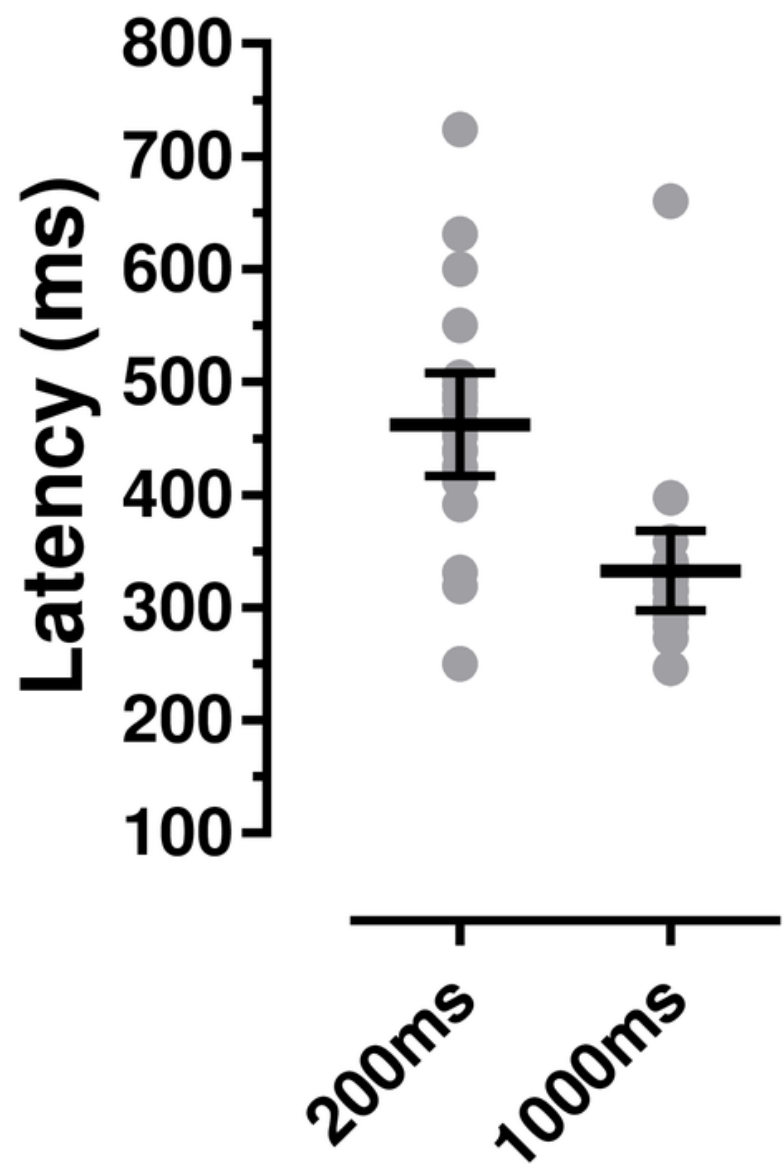

b. Error Rate
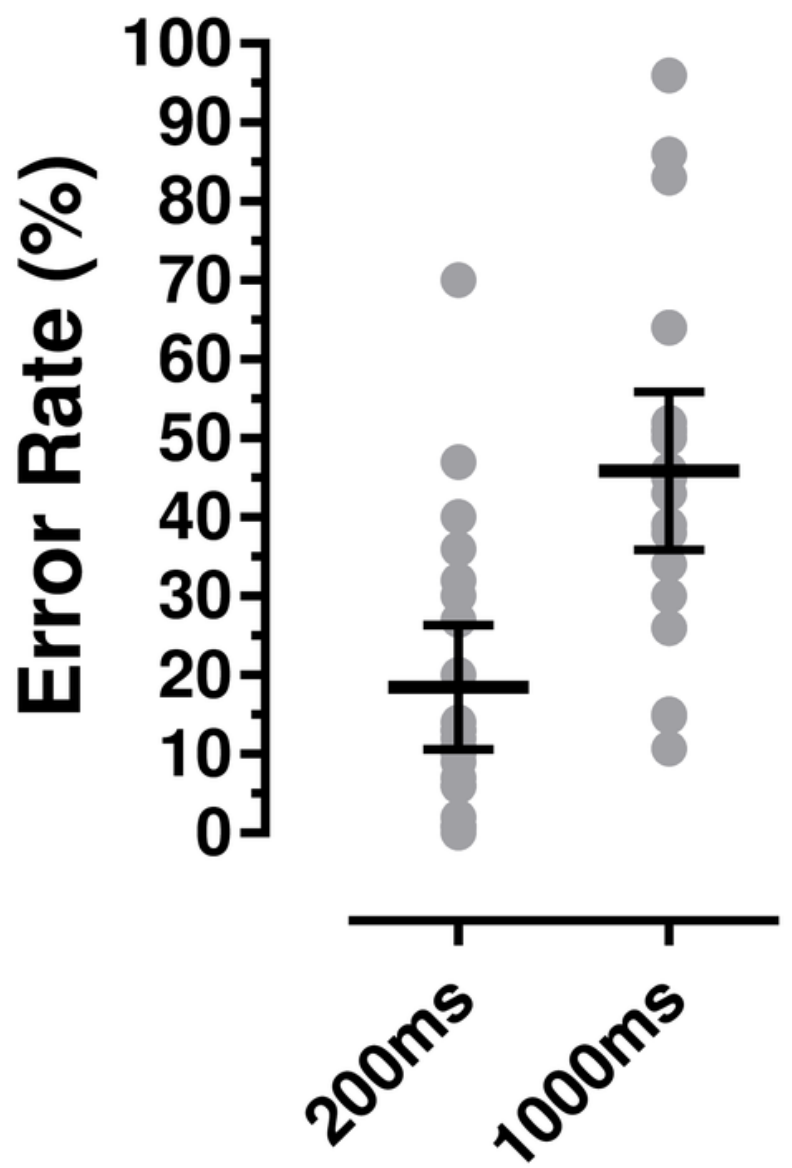

Target Display Duration 
Figure 3

Comparison of MDOR latency in old and young participants.

Comparison of latency between old and young groups. Mean ( $\pm 95 \% \mathrm{Cl}$ 's) is shown. (A) Raw latency. (B) Corrected latency. Latency in the calibration task is subtracted from each participant's MDOR latency.

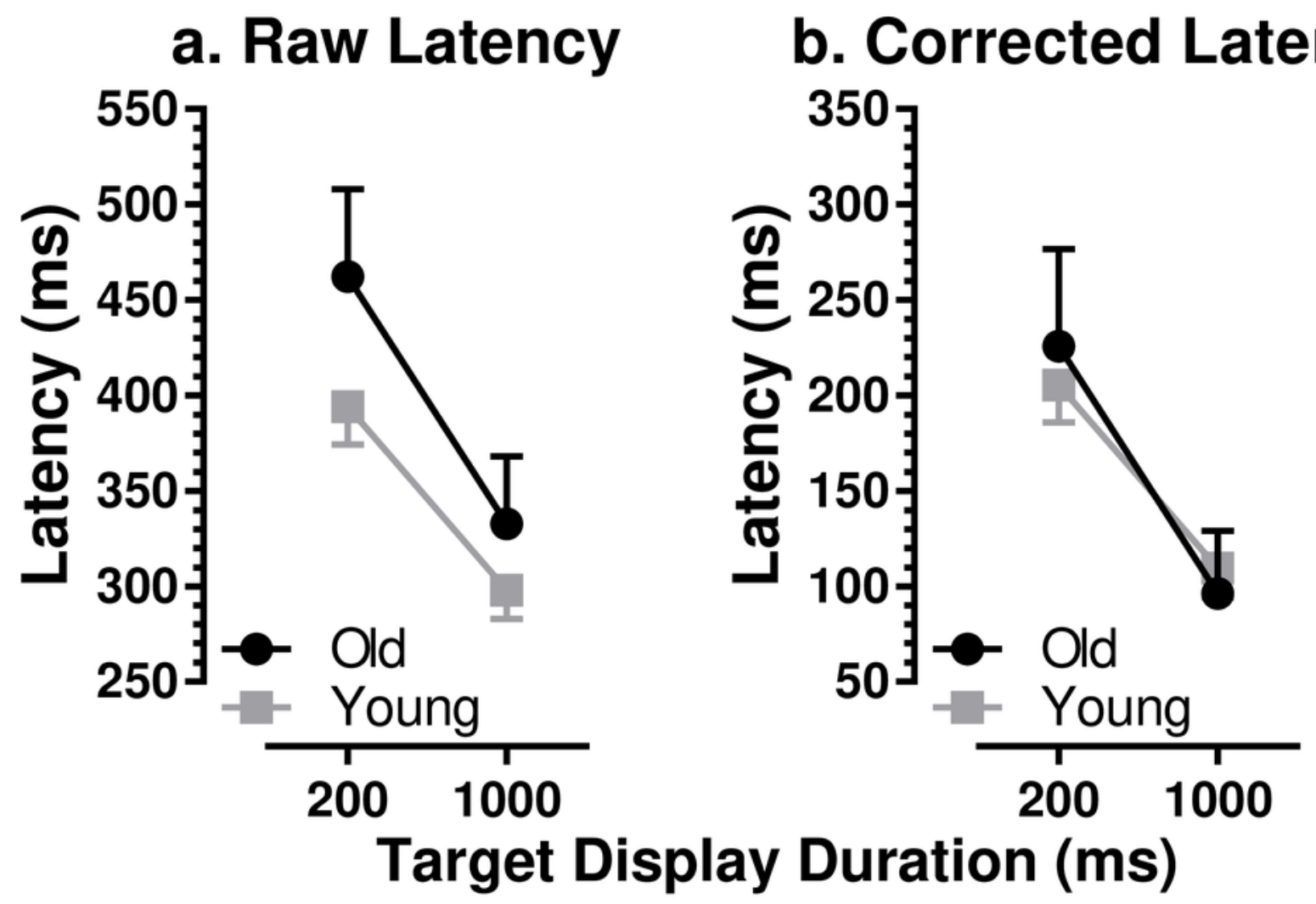


Figure 4

Comparison of error rate in old and young participants

Mean $( \pm 95 \% \mathrm{Cl})$ MDOR error rate (\%) in old and young participant groups. 


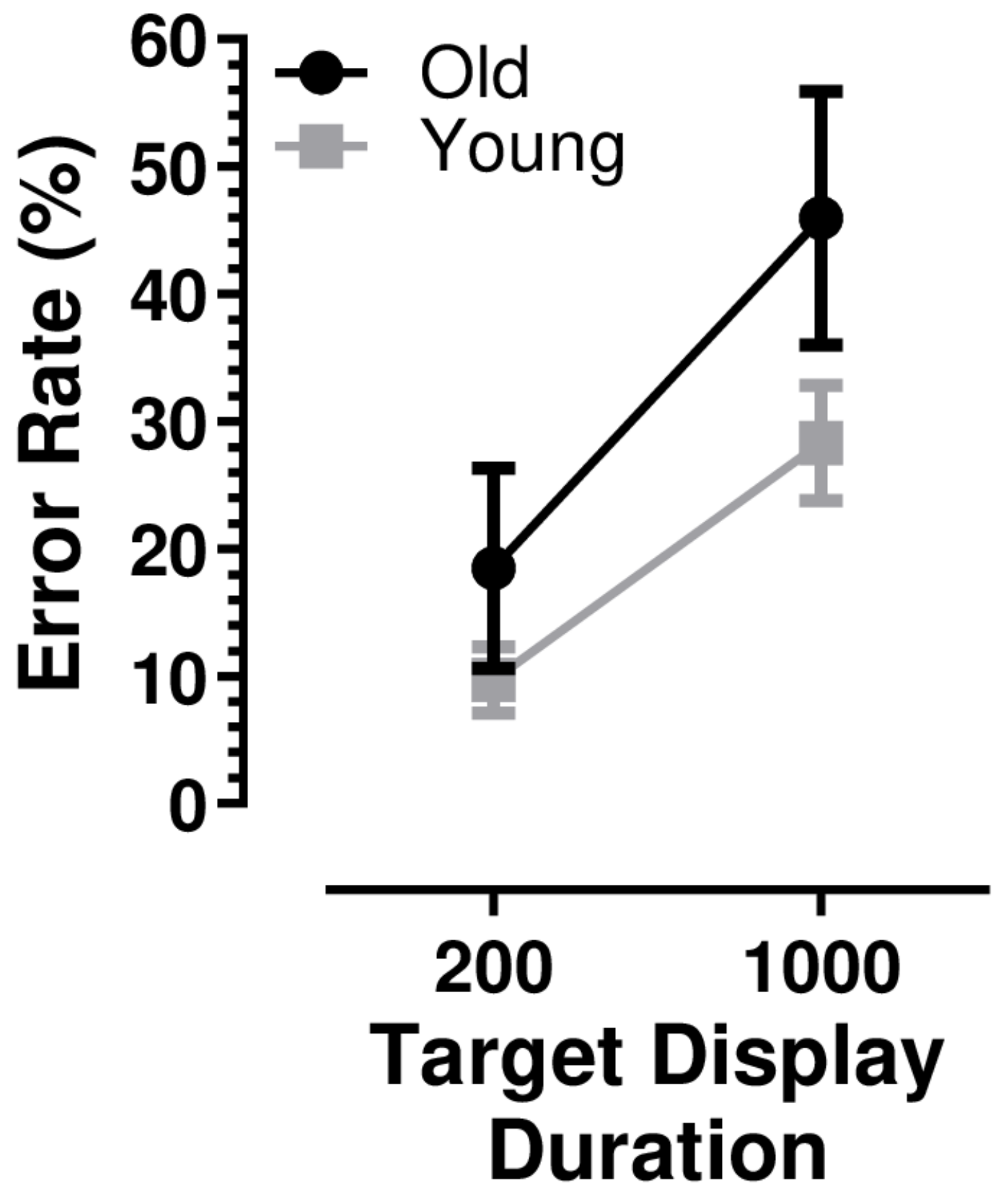




\section{Figure 5}

Average distributions of response timings for old and young participant groups.

Average distributions are shown for old $(a, b)$ and young $(c, d)$ participant groups. Bin

width $=50 \mathrm{~ms}$. For each participant, the \% frequency distribution for responses was calculated.

The mean $( \pm 95 \% \mathrm{Cl})$ was then calculated for each bin across participants in each group. The black central line is plotted through the each mean bin value, while grey lines show $\pm 95 \% \mathrm{Cl}$. Target onset is at -200 for 200ms TDD $(a, c)$ and -1000ms for 1000ms TDD (b,d); target offset (the go signal) is at $0 \mathrm{~ms}$ (vertical dotted line). Arrows mark peak bins in each distribution for both errors and correct responses. Grey regions mark the bin ranges over which error rate was recalculated. 

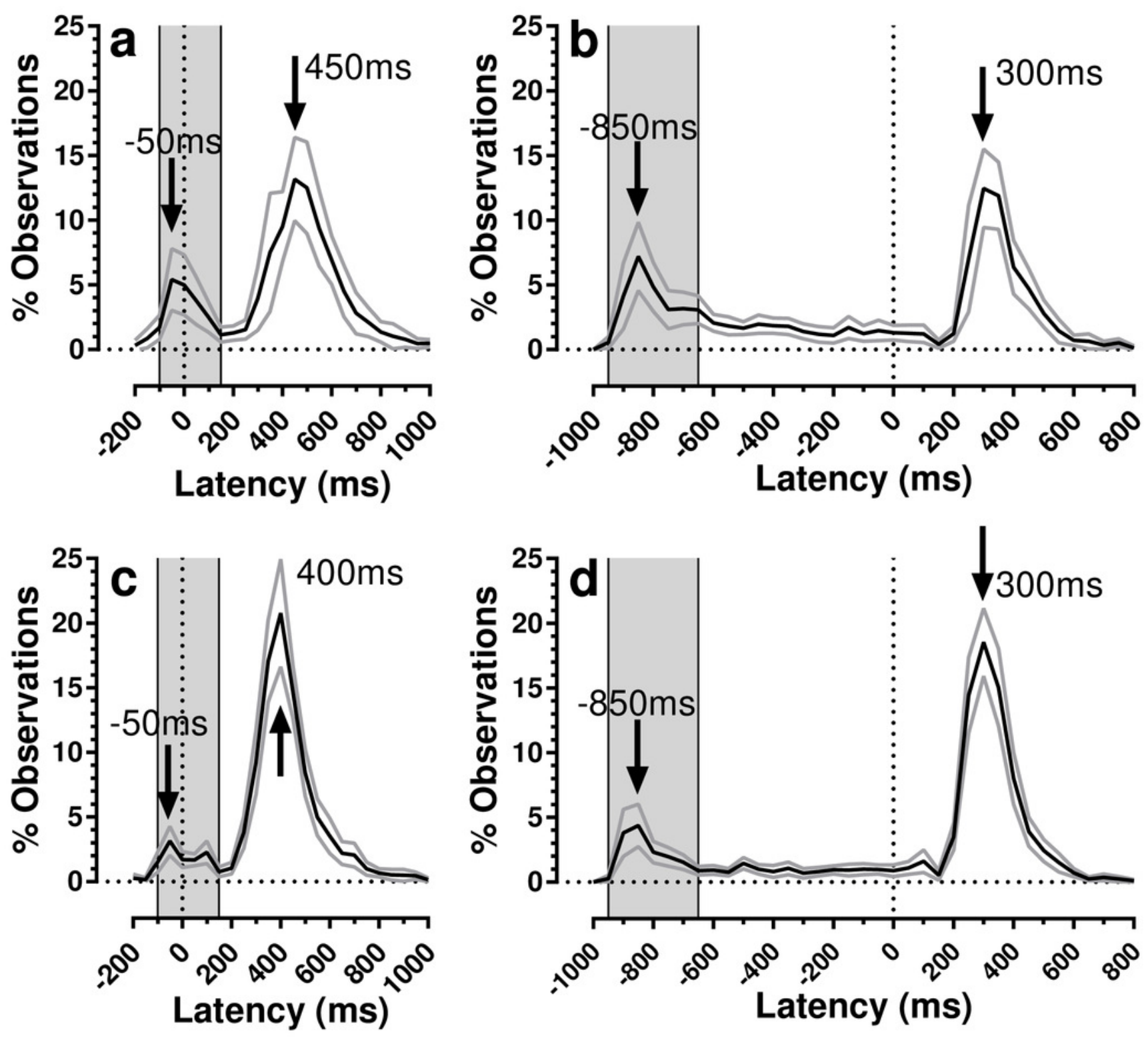